\title{
An Improved Algorithm for Sequence Pair Generation
}

\author{
Mingxu Huo and Koubao Ding* \\ Dept. of Information and Electronic Engineering, Zhejiang University, \\ Hangzhou 310027, P.R. China \\ \{huomingxu, dingkb\}@zju.edu.cn
}

\begin{abstract}
Sequence Pair is an elegant representation for block placement of IC design, and the procedure to generate the SP from an existing placement is necessary in most cases. An improved generation algorithm is proposed instead of the existing methods that are either difficult or inefficient to be implemented. The algorithm simplifies the definition of relation between blocks and avoids employing complicated graph operations. The time complexity of the algorithm is $\mathrm{O}\left(\mathrm{n}^{2}\right)$ and can be reduced to $\mathrm{O}(\mathrm{n} \log \mathrm{n})$, where $\mathrm{n}$ is the number of blocks. The experimental results of the algorithm show its superiority in running time.
\end{abstract}

\section{Introduction}

The floorplanning and block placement problems become increasingly important in physical design of Integrated Circuits (IC), where floorplanning can be regarded as placement with soft module blocks. Such problems are usually solved in two phases, i.e., the initial constructive phase, and iterative improvement one [1]. They are complex combinatorial optimization problems and most of their sub-problems are NP-Complete or NP-Hard [2]. Therefore heuristic approaches such as Simulated Annealing (SA) algorithm [3] are widely used to generate good layouts at the iterative stage, where representations of the placement is one of the crucial factors in evaluation of the costs.

In contrast with the so-called flat or absolute representations where the blocks are specified in terms of absolute coordinates on a plane without grids, a large number of representations of geometrical topological relations of blocks were proposed, e.g., slicing [4], mosaic [5], compacted [6] and $\mathrm{P}^{*}$-admissible representations [2, 7, 8, 9]. The $\mathrm{P}^{*}$-admissible representations can represent the most general floorplans and contain a complete structure for searching an optimal solution, among which the Sequence Pair (SP) is most favored and widely researched recently.

The SP related efforts with SA algorithms in the literature usually randomly generate an initial SP and then pack it to evaluate its cost. However, Force Directed Relaxation [10, 11] and other analytical algorithms that have been studied for a long time can construct better placements than the random ones. In addition, with the occurrence of incremental physical design [12] or Engineering Change Orders (ECO), it is imperative to find an effective and efficient approach to generate the SP from an arbitrary existing placement as an initial configuration.

\footnotetext{
${ }^{*}$ Corresponding author.
} 
The original method to generate SP is "Gridding" [2], which is so complicated that it can hardly be implemented and the time complexity is assumed to be $\mathrm{O}\left(\mathrm{n}^{3}\right)$, where $\mathrm{n}$ is the number of blocks. The generation procedure in Parquet [13] uses dynamic programming algorithm to find transitive closure graphs (TCG), which runs in $\mathrm{O}\left(\mathrm{n}^{2}\right)$ time. In [14], Huo and Ding proposed a much faster generation method and an algorithm that runs much faster than the TCG algorithm from Parquet. As we know, the time complexity of traversing a graph is $\mathrm{O}\left(\mathrm{n}^{2}\right)$ or $\mathrm{O}(\mathrm{n}+\mathrm{e})$, where $\mathrm{e}$ is the number of edges, although it was reported (without proof or experimental results) by Kodama et. al. that the time complexity of the proposed "Fast-Gridding" algorithm by means of tracing a constraint graph in [15] is $O(n \log n)$.

In this paper, an improved algorithm to generate SP is proposed, which determines the position of each block on a generation plane based on the relations of every two blocks, and the time complexity is $\mathrm{O}\left(\mathrm{n}^{2}\right)$. A faster $\mathrm{O}(\mathrm{n} \log \mathrm{n})$ algorithm is also proposed.

\section{Sequence Pair}

The topological relations of any two non-overlap module blocks are horizontal and vertical, i.e., left to, right to, above and below [16], as shown in Fig. 1. Diagonal relations can be simply degenerated by preferring horizontal relations to vertical ones, as in Fig. 1(c) where $b_{i}$ is assumed to be left to $b_{j}$, unless there is a chain of vertical relations, which is considered as a vertical relation, as shown in Fig. 1(d). For example, in Fig. 2 block 8 is both left to and above block 3, but there is a vertical relation chain of them, i.e., block 8 is above block 6 and block 6 above block 3, so block 8 is said to be above block 3 instead of left to it. Such relations cannot be determined by just check two blocks, so that many procedures calculate indirect relations from the transitive closure of immediate relations, which is usually timeconsuming.

An HV-Relation-Set (HVRS) for a set of blocks is a set of horizontal or vertical relations for all block pairs [17], and a Feasible-HVRS involves all the relations of blocks excluding non-realizable relations, e.g. $\left\{b_{i}\right.$ is left to $b_{j}, b_{j}$ is left to $b_{k}, b_{k}$ is left to $b_{i}$ \} is not a Feasible-HVRS for blocks $b_{i}, b_{j}$ and $b_{k}$.

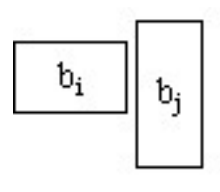

(a)

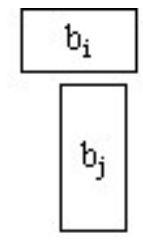

(b)

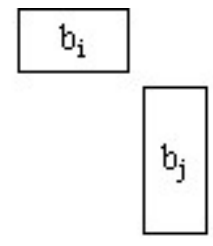

(c)

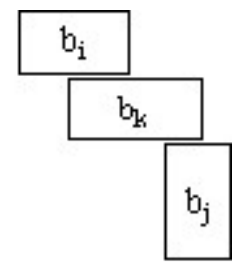

(d)

Fig. 1. Possible relations between two blocks $b_{i}$ and $b_{j}$. (a) $b_{i}$ is left to $b_{j}$; (b) $b_{i}$ is above $b_{j}$; (c) $b_{i}$ is diagonal to $b_{j}$ and assumed to be left to $b_{j}$; (d) $b_{i}$ is diagonal to $b_{j}$ but a vertical relation chain exists between $b_{i}$ and $b_{j}$, i.e., $b_{i}$ is above $b_{k}$ and $b_{k}$ is above $b_{j}$, so $b_{i}$ is above $b_{j}$. 
A Sequence Pair (SP) is an ordered pair of sequences $\Gamma_{+}$and $\Gamma_{-}$, each of which is a sequence of $n$ block names [2], e.g., the SP from Fig. 2 is $\left(\Gamma_{+}, \Gamma_{-}\right)=(154806273$, 012453687). A sequence pair corresponds to a Feasible-HVRS as follows:

$-b_{i}$ is left to $b_{j}\left(b_{j}\right.$ is right to $\left.b_{i}\right)$ : if $\left(\Gamma_{+}, \Gamma_{-}\right)=\left(\ldots b_{i} \ldots b_{j} \ldots, \ldots b_{i} \ldots b_{j} \ldots\right)$

$-b_{i}$ is below $b_{j}\left(b_{j}\right.$ is above $\left.b_{i}\right)$ : if $\left(\Gamma_{+}, \Gamma_{-}\right)=\left(\ldots b_{j} \ldots b_{i} \ldots, \ldots b_{i} \ldots b_{j} \ldots\right)$

The time complexity of the original evaluation procedure "Packing" is $O\left(n^{2}\right)[2]$, and it was later sped up to $\mathrm{O}(\mathrm{n} \log \log \mathrm{n})$ [18].

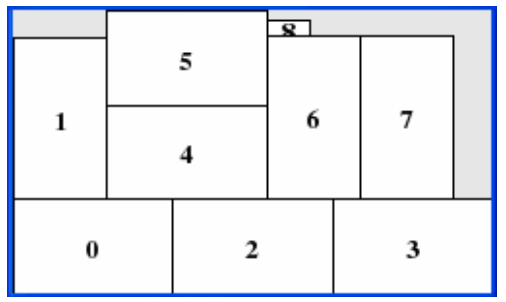

Fig. 2. A placement of the MCNC apte benchmark with SP (1 54806273,012453687 )

\section{Generation Algorithms}

\subsection{Algorithm Embedding}

According to the generation method Embedding from [14], algorithm Embedding can be proposed naturally. To determine the Feasible-HVRS of $\mathrm{n}$ blocks, each block needs to be compared with every other $n-1$ blocks, which implies the $\mathrm{O}\left(n^{2}\right)$ time complexity. However, not all the comparisons are necessary and some of them can be removed to accelerate the procedure, e.g., if the information of $b_{i}$ above $b_{j}$ and $b_{j}$ above $b_{k}$ is acquired, $b_{i}$ is absolutely above $b_{k}$.

\section{Resolve relations and ordering criteria in sequences}

The orders of blocks in both sequences can be determined from relations of blocks $b_{i}$ and $b_{\mathrm{j}}$, as follows:

- $b_{i}$ is before $b_{j}\left(b_{j}\right.$ is behind $\left.b_{i}\right)$ in $\Gamma_{+}$: if $b_{i}$ is left to $b_{j}$ or above $b_{j}$

- $b_{i}$ is before $b_{j}\left(b_{j}\right.$ is behind $\left.b_{i}\right)$ in $\Gamma_{-}$: if $b_{i}$ is left to $b_{j}$ or below $b_{j}$

$-b_{i}$ is before $b_{k}$ in either sequence: if $b_{i}$ is before $b_{j}$ and $b_{j}$ is before $b_{k}$.

The relations of any two blocks can be just determined by their locations and sizes. Here we ignore the vertical chain and if $b_{i}$ and $b_{j}$ have both horizontal and vertical relations. We simply assume their relation is horizontal.

\section{Sort the blocks according to their $\mathrm{x}$ coordinates}

The order of blocks to be embedded must be determined, in order to eliminate ambiguities and achieve a correct result. In this algorithm, we sort the blocks 
according to their $\mathrm{x}$ coordinates with introspective sorting and selection algorithms that performs $\mathrm{O}(\mathrm{n} \log \mathrm{n})$ time complexity in worst cases as discussed in [19].

\section{Insert block names into linked lists according to the sorted order}

Two linked lists listx and listy are used to represent the $\Gamma_{+}$and $\Gamma_{-}$respectively, which are generated separately by inserting the block names. To insert block $b_{i}$ into a list, we traverse the list from its header, check the relations of $b_{i}$ with every visited block $b_{j}$, and stop to insert $b_{i}$ before $b_{j}$, if it is found that $b_{j}$ should be behind $b_{i}$ in the list. However, if no such block exists, $b_{i}$ is appended to list because it should be current list tail.

Program section of Embedding for in C++ (only for listx)

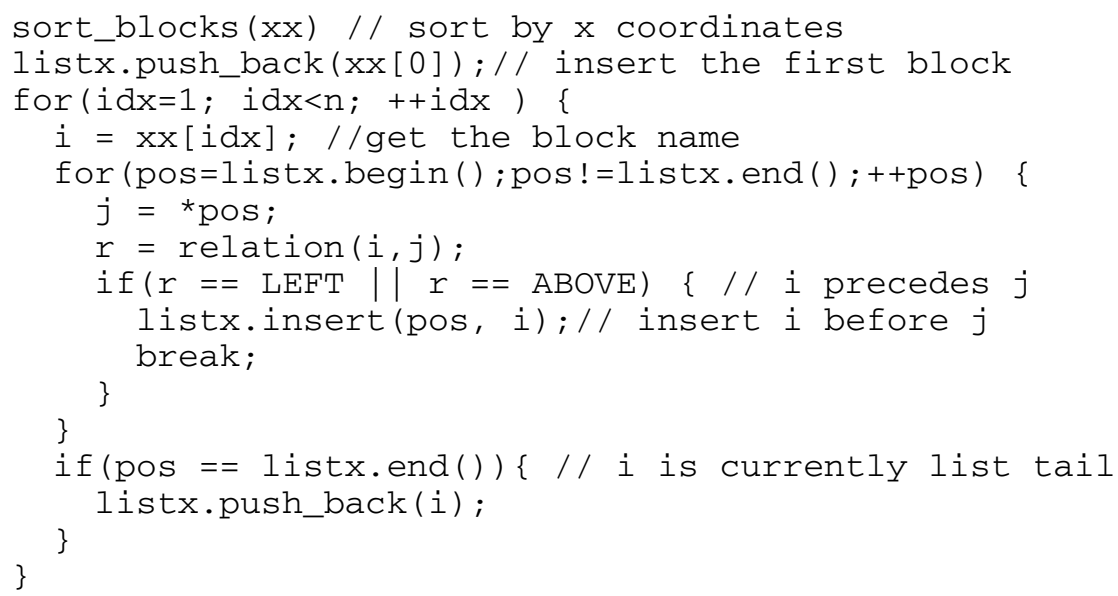

The procedure "relation" in the algorithm compares block $b_{i}$ and block $b_{j}$ only, so it runs for constant time. The time complexity of the outer for loop is $O(n)$, so is the inner one. And thus the total time complexity of algorithm Embedding is $\mathrm{O}\left(\mathrm{n}^{2}\right)$.

Sometimes a placement may correspond to multiple sequence pairs, e.g., the placement in Fig. 2 has another sequence pair (1 54806723,012345678 ) as well as the one discussed in this paper. We pack the generated SP again and check whether the locations of all the blocks in the new placement match those in the old one, in order to validate the result.

A sequence pair obtained by Embedding imposes horizontal relation on any pair of blocks only when the relation of the pair cannot be coded as vertical.

\section{Lemma. Embedding is correct when vertical relation chains exists.}

Proof: Suppose the relation of $b_{i}$ and $b_{j}$ is diagonal and there exists a chain of vertical relations between them. Without loss of generality, $b_{i}$ is supposed to be left to and above $b_{j}$, and only one block $b_{k}$ is in the chain, i.e., $b_{i}$ is above $b_{k}$ and $b_{k}$ is above $b_{j}$, as illustrated in Fig. 1 (d). If the relation of $b_{i}$ and $b_{j}$ is checked first, the incorrect result will be returned, i.e., $b_{i}$ is left to $b_{j}$, according to the ordering criteria. Now that the relation of $x$ coordinates of the three blocks is $x_{i}<x_{k}<x_{j}$, and the order to embed blocks is according to their $x$ coordinates, $b_{k}$ is embedded in advance of $b_{j}$ and the correct vertical relation of $b_{i}$ and $b_{k}$ is obtained first. Accordingly, the correct vertical 
relation of $b_{k}$ and $b_{j}$ is obtained later, so the relation of $b_{i}$ and $b_{j}$ is implicated correctly, i.e., a vertical one, instead of being mistakenly resolved.

\section{Theorem. The Sequence Pair generated by algorithm Embedding is correct.}

Proof: If no diagonal relations exist, the relation of every two blocks is unique and the orders of blocks in both sequences can be correctly determined. If diagonal relations exist but there are no chains of vertical relations, since we regard such diagonal relations as horizontal ones, the relation of every pair of blocks is also unique and the result is correct. The last case is there are both diagonal relations and vertical relation chains in a placement. According to the preceding Lemma, the algorithm is correct too. Since the relations of every pair of blocks are determined without ambiguity, the order in either sequence of SP is correct.

\subsection{Reducing Running Time to $\mathbf{O}(\mathbf{n} \log n)$}

Since both $\Gamma_{+}$and $\Gamma_{\text {. }}$ are ordered sequences, we can use balanced binary search trees to store them. Make use of two binary trees treex and treey to represent $\Gamma_{+}$and $\Gamma_{\text {. }}$ respectively, and generate treex and treey separately by inserting the blocks into the trees. We name this algorithm LogAlgo.

Program section of LogAlgo algorithm in C++ (for treex only)

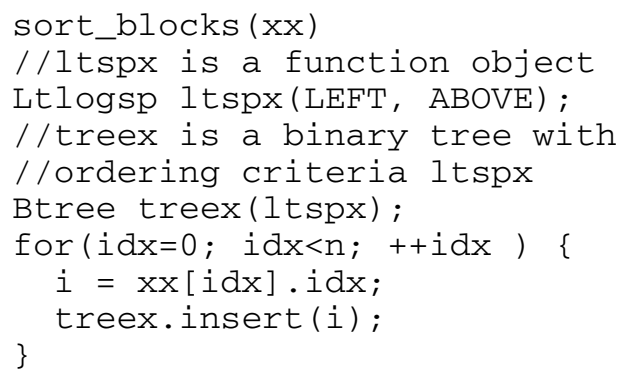

Class Ltlogsp invokes procedure relation $(i, j)$ that functions the same with the one of algorithm Embedding when a comparison of two blocks $b_{i}$ and $b_{j}$ is needed. The insertion operation of Btree includes searching on a binary tree and keeping it balanced, whose time complexity is $O(\log n)$. There are $n$ blocks to be inserted to the tree in the loop, so the total time complexity is now reduced to $O(n \log n)$ from $O\left(n^{2}\right)$.

\subsection{Experimental Results}

After sorting, the order of blocks of the placement in Fig. 1 to be embedded is: 0145

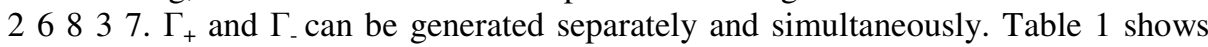
the running process of the algorithm Embedding to generate the listx from the placement in Fig. 1 (the results for listy and for other benchmarks is omitted), and the number of comparisons is 34. Table 2 shows the process of algorithm LogAlgo, with 20 comparisons. Fig. 3 demonstrates the balanced binary tree treex when block 3 and block 7 is inserted respectively. 
Table 1. Run of the algorithm after inserted block 0 in list listx. A, B, L and R stand for relations between two blocks of above, below, left to and right to respectively. List listx is the list before (without block name in parenthesis) and after intertion of current block.

\begin{tabular}{lllrll}
\hline$\#$ & Comparisons & ListX & $\#$ & Comparisons & ListX \\
\hline $\mathbf{1}$ & A0 & $(1) 0$ & $\mathbf{6}$ & R1, R5, R4, R0, A2 & 1540 (6) 2 \\
$\mathbf{4}$ & R1, A0 & $1(4) 0$ & $\mathbf{8}$ & R1, R5, R4, R0, A6 & 1540 (8) 62 \\
$\mathbf{5}$ & R1, A4 & $1(5) 40$ & $\mathbf{3}$ & R1, R5, R4, R0, R8, & $1540862(3)$ \\
& & & & B6, R2 \\
$\mathbf{2}$ & R1, B5, B4, R0 & $1540(2)$ & $\mathbf{7}$ & R1, R5, R4, R0, R8, \\
& & & & R6, R2, A3 \\
\hline
\end{tabular}

Table 2. Run of the algorithm after inserted block 0 in Btree treex. The inorder traversal results of treex are listed in column TreeX.

\begin{tabular}{llllll}
\hline$\#$ & Comparisons & TreeX & $\#$ & Comparisons & TreeX \\
\hline $\mathbf{1}$ & A0 & $(1) 0$ & $\mathbf{6}$ & R4, R0, A2 & $1540(6) 2$ \\
$\mathbf{4}$ & A0, R1 & $1(4) 0$ & $\mathbf{8}$ & R4, A6, R0 & $1540(8) 62$ \\
$\mathbf{5}$ & A4, R1 & $1(5) 40$ & $\mathbf{3}$ & R4, B6, R2 & $1540862(3)$ \\
$\mathbf{2}$ & B4, R0 & $1540(2)$ & $\mathbf{7}$ & R4, R6, R2, A3 & $1540862(7) 3$ \\
\hline
\end{tabular}

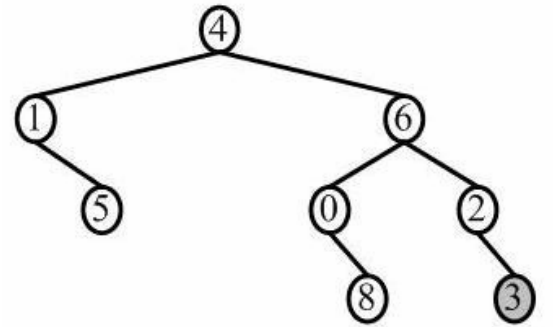

(a)

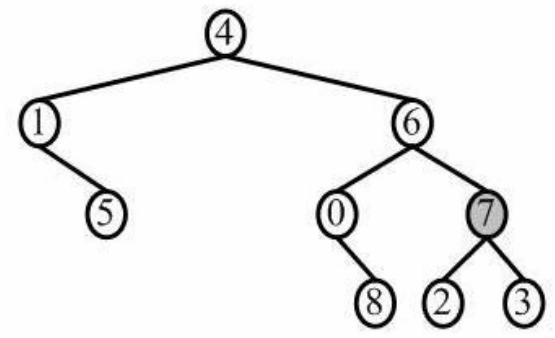

(b)

Fig. 3. the Btree treex (a) after block 3 and (b) block 7 was inserted, respectively. The newly inserted node is in shade. Please note that after block 7 was inserted, the tree rotated to keep balanced.

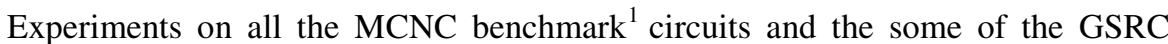
benchmark $^{2}$ circuits are performed in comparison with the generation routine from Parquet [13] on running time. We first generate the placements of the benchmarks with the Simulated Annealing algorithm using the LCS evaluation algorithm [18], and save the placements and their corresponding sequence pairs. Then we take the placements as input and generate their sequence pairs as output with the programs respectively. The accumulative running time of the generation 1000 times are listed in

\footnotetext{
${ }^{1}$ See: http://www.cse.ucsc.edu/research/surf/GSRC/MCNCbench.html

${ }^{2}$ See: http://www.cse.ucsc.edu/research/surf/GSRC/GSRCbench.html
} 
Table 3. It can be seen that our algorithms runs much faster than the routine from Parquet, and the more complex the placement is, the more speed improvement our program achieves. In addition, as the number of blocks grows larger than 33, the LogAlgo algorithm with $\mathrm{O}(\mathrm{n} \log \mathrm{n})$ time complexity outperforms the Embedding evidently. When there are less than 33 blocks, additional time is needed for the LogAlgo algorithm to keep the tree balanced.

Table 3. Running time in seconds of our programs and Paquet on MCNC and GSRC benchmarks

\begin{tabular}{lllll}
\hline Benchmark & block \# & Parquet & Embedding & LogAlgo \\
\hline apte & 9 & 0.0580 & 0.0100 & 0.0120 \\
xerox & 10 & 0.0740 & 0.0110 & 0.0130 \\
hp & 11 & 0.0840 & 0.0120 & 0.0130 \\
ami33 & 33 & 0.7369 & 0.0470 & 0.0440 \\
ami49 & 49 & 1.8127 & 0.0780 & 0.0670 \\
n50a & 50 & 1.7257 & 0.0830 & 0.0670 \\
n100a & 100 & 7.4579 & 0.2570 & 0.1380 \\
n200a & 200 & 35.8016 & 0.8989 & 0.2940 \\
n300a & 300 & 92.4549 & 1.9057 & 0.4349 \\
\hline
\end{tabular}

\section{Conclusions}

In this paper, we introduce an improved algorithm named Embedding to generate Sequence Pair, which is easier to be implemented than the original "Gridding" method [2], is more elegant than the graph-based algorithm "Fast-Gridding"[15] and runs faster than the existing implementation from Parquet. The algorithm uses linked lists to store the block sequence, whose time complexity is $\mathrm{O}\left(\mathrm{n}^{2}\right)$. We also proposed an algorithm LogAlgo based on balanced binary search trees that costs $O(n \log n)$ running time. When a placement is composed with more than 33 blocks the LogAlgo reveals its superiority.

\section{References}

1. Goto, S., An efficient algorithm for the two-dimensional placement problem in electrical circuit layout,IEEE Trans. On Circuits and Systems, (1998), 28(1): 12 - 18

2. Murata, H., et al., Rectangle-packing-based module placement. 1995 IEEE/ACM ICCAD. Digest of Technical Papers, (1995):. 472-479.

3. Kirkpatrick, S., C.D. Gelatt, and M.P. Vecchi, optimization by simulated annealing. Science, 1983. 220(4598): (1983) 671-680.

4. Otten, R.H.J.M., Automatic floorplan design. ACM IEEE 9th DAC Proceedings, (1982): 261-267. 
5. Hong, X.L., et al., A non-slicing floorplanning algorithm using corner block list topological representation, in 2000 IEEE Asia-Pacific Conference On Circuits And Systems - Electronic Communication Systems. (2000). 833-836.

6. Pei-Ning, G., C. Chung-Kuan, and T. Yoshimura, An O-tree representation of non-slicing floorplan and its applications. Proceedings 1999 DAC, (1999): 268-273.

7. Nakatake, S., et al., Module packing based on the BSG-structure and IC layout applications. IEEE TCAD Of Integrated Circuits And Systems, (1998). 17(6): 519-530.

8. Jai-Ming, L. and C. Yao-Wen, TCG: a transitive closure graph-based representation for non-slicing floorplans. Proceedings of the 38th DAC, (2001): 764-769.

9. Lin, J.M. and Y.W. Chang, TCG-S: Orthogonal coupling of $\mathrm{P}^{*}$-admissible representations for general floorplans, in 39th DAC, Proceedings. (2002). 842-847.

10. M. Sarrafzadeh and M. Wang, NRG: Global and detailed placement, Proc. IEEE ICCAD, 1997. (1997):532-537

11. N. Quinn, and M. Breuer, A force-directed component placement procedure for printed circuit boards, IEEE Trans. CAS, vol.CAS-26, no.1979(6):.377-388

12. Cong, J. and M. Sarrafzadeh, Incremental physical design. Proceedings International Symposium on Physical Design, 2000. ISPD-2000, (2000): 84-92.

13. Adya, S.N. and I.L. Markov, Fixed-outline floorplanning through better local search. Proceedings 2001 IEEE ICCD: VLSI in Computers and Processors. 2001, (2001): 328-334.

14. Huo, MX and Ding, KB, A quick generation method of sequence pair for block placement, COMPUTATIONAL SCIENCE - ICCS 2005, PT 3 3516: 954-957

15. Kodama, C., Fujiyoshi, K. and Koga, T., A novel encoding method into sequence-pair,. Proceedings ISCAS 2004, 2004 (5): 329 - 332

16. Onodera, H., Y. Taniguchi, and K. Tamaru, Branch-and-bound placement for building block layout. 28th ACM/IEEE DAC. Proceedings 1991, (1991): 433-439.

17. Murata, H., et al., A mapping from sequence-pair to rectangular dissection. Proceedings of the ASP-DAC '97. 1997, (1997): 625-633.

18. Xiaoping, T. and D.F. Wong, FAST-SP: a fast algorithm for block placement based on sequence pair. Proceedings of the ASP-DAC 2001. 2001, (2001): 521-526.

19. D. R. Musser, "Introspective Sorting and Selection Algorithms", Software Practice and Experience, 1997, 27(8):983 